DOI: http://doi.org/10.21698/simi.2018.ab19

\title{
THE PHOTOLYSIS OF EPIRUBICIN IN AQUEOUS MEDIA
}

\author{
Mirabela Soare, Petruta Oancea, Adina Raducan
}

Department of Physical Chemistry, Faculty of Chemistry, University of Bucharest, 4-12

Elisabeta Boulevard, Bucharest, petrutaoancea73@yahoo.com, Romania

Keywords: epirubicin, photochemical stability, primary process

\section{Introduction}

Anthracyclines are considered the most effective drugs in the treatment of cancer. Epirubicin (Epi) is an anthracycline drug used in chemotherapy. Epirubicin was shown to be as effective as doxorubicin, but with less cardiac toxicity at comparable doses. The great majority of therapeutic substances are white in appearance, meaning that they do not absorb light in the visible region, but they may absorb in the UV region as a consequence of their chemical structure. Epirubicin is colored, so it absorbs the visible light, being susceptible to photodegradation. The evaluation of the photochemical stability of drugs and drug products is an essential component of the formulation development process in the pharmaceutical industry.

\section{Materials and methods}

The UV-vis spectra of drug were recorded at room temperature from 200 to $700 \mathrm{~nm}$ using a Jasco V-530 spectrophotometer with a spectrometric quartz cell $(1 \mathrm{~cm}$ path length). The maximum absorbance wavelength $\left(\lambda_{\max }\right)$ of Epirubicin is $483 \mathrm{~nm}$.

Photostability of epirubicin solutions was testated in the presence of either UltraViolet or Visible light. The source used for the UV irradiations was a low-pressure mercury lamp which emits wavelength at $254 \mathrm{~nm}$. For visible range were used medium-pressure mercury lamps with power 150 and $250 \mathrm{~W}$, respectively. The lamp equipped with an UV-VIS radiation transparent glass water cooling jacket is immersed in the centre of the reactor containing the drug solution.

\section{Results and conclusions}

Solutions of Epirubicin were irradiated with UV and Visible radiations in order to evaluate its photostability. In Figure 1 are presented evolution of absorption spectra for $7.6 \times 10^{-5} \mathrm{M}$ initial concentration by visible irradiation. The effect of initial EPI concentrations $\left(1 \times 10^{-5} \mathrm{M}-4 \times 10^{-4} \mathrm{M}\right)$ on the photochemical degradation has been investigated and the pseudo-first order rate constant and quantum yield were calculated. These parameters varied with the initial concentration, decreasing as the concentration was increased. An explanation to this behavior is "self-qenching" processes, where the excited states are deactivated through collisions with the molecules in the ground state. 


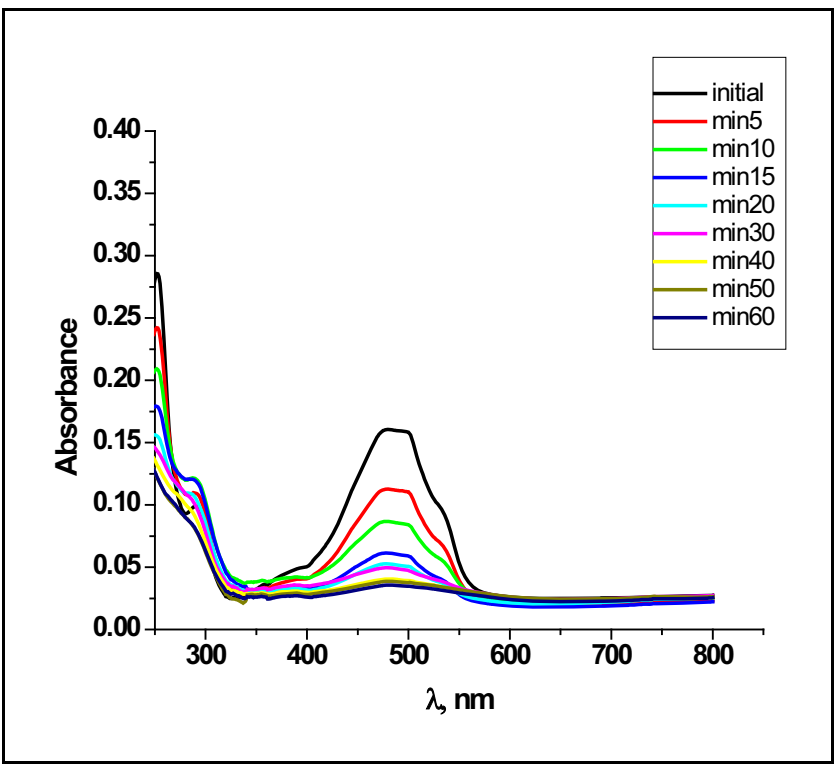

Figure 1. The evolution of absorption spectrum with the exposure time

Our experimental results are correlated with the available literature information leading us to the following mechanism of the primary process (Figure 2):

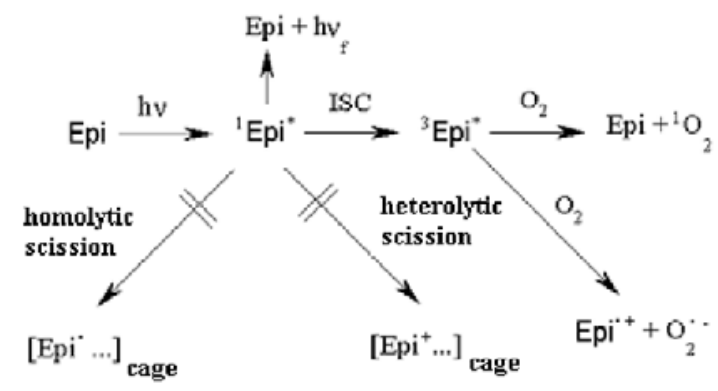

Figure 2. The primary photochemical process of Epi photolysis

Through the light absorption process, the electronically excited state ${ }^{1} \mathrm{Epi}^{*}$ is generated. This can be deactivated by physical processes (radiative or non-radiative deactivation) or thermal chemical reaction. This reaction scheme involves both the singlet and triplet excited species. The excited singlet state ${ }^{1} \mathrm{Epi}^{*}$ may be deactivated by fluorescence emission, homolytic or heterolytic bond scission and intersystem crossing (ISC) process with the appearance of ${ }^{3} \mathrm{Epi}^{*}$. The low quantum yields determined experimentally show that only the last process takes place whereas the recombination in the solvent cage leading to the initial molecule occurs with high probability. The presence of $\mathrm{O}_{2}$ in the studied system play an important role, also supported by the results obtained in the presence and absence of this $\left(\mathrm{k}_{\mathrm{Ar}}<\mathrm{kO2}_{\mathrm{a}}\right.$-atm $<$ ko2-bubbled). Kinetic studies suggest that epirubicin in aqueous solution is more susceptible to degradation by exposure to Visible light than UV light. 